\title{
Hyperfluorescent dots in the ciliary body band in patients with granulomatous uveitis
}

\author{
RYOZO KIMURA \\ From the Department of Ophthalmology, School of Medicine, Tohoku University, 1-1 Seiryo-cho, \\ Sendai 980 , Japan
}

SUMMARY Fluorescein gonioangiography was performed in 4 eyes of 4 patients with ocular sarcoidosis and in an eye of a patient with presumptive tuberculous anterior uveitis. Small discrete whitish lesions in the ciliary body band near the iris root showed hyperfluorescence shortly after fluorescein injection. These hyperfluorescent dots seemed to be pathognomonic changes in cases with granulomatous uveitis.

In 1965 Eisner $^{1}$ observed the anterior chamber angle after fluorescein injection and reported that staining of the ciliary body band occurred in cases of cyclitis. In 1980 the author ${ }^{2}$ presented gonioangiograms which showed vigorous leakage of the dye from the angle recess into the anterior chamber in a case of Behçet's disease during an attack of uveitis. To the best of my knowledge there are no studies of fluorescein gonioangiography in cases of granulomatous uveitis. The purpose of this communication is to report gonioangiographic findings in such cases.

\section{Patients and methods}

The same method of fluorescein gonioangiography as previously described ${ }^{2}$ was used in this study. Four eyes of 4 patients with ocular sarcoidosis and one eye of a patient with presumptive tuberculous anterior uveitis were selected from our clinic for this study. All the patients had a complete eye examination. Those with a history of ocular surgery and ocular trauma and with abnormal intraocular pressure were excluded. In all 4 patients with ocular sarcoidosis the diagnosis was established by a biopsy of either conjunctival follicles or scalene nodes.

\section{Case reports}

\section{OCULAR SARCOIDOSIS}

Case 1. A 21-year-old male was diagnosed as having bilateral hilar lymphadenopathy and was referred to our clinic. Vision was 20/20 without correction in both eyes. Biomicroscopy showed neither keratic

Correspondence to Dr R. Kimura. precipitates nor flare in the anterior chamber in both eyes. The iris was normal in appearance in both eyes. He had complained of no eye symptoms. However, ophthalmoscopy revealed 'candle wax' drippings along the peripheral retinal vessels in both eyes. The intraocular pressure was $12 \mathrm{mmHg}$ in the right eye and $11 \mathrm{mmHg}$ in the left. Gonioscopy revealed small whitish lesions in the ciliary body band near the iris root associated with discrete gelatinous nodular lesions in the filtration angle in both eyes. Fluorescein gonioangiography (Fig. 1) in the lower nasal quadrant of the right eye was performed, and it showed soon after the fluorescein injection a hyperfluorescent dot corresponding to the whitish lesion in the ciliary body band. The gelatinous nodular lesions in the filtration angle showed relative hyperfluorescence in the late phase of the angiography. A tuberculin skin test was negative, and the diagnosis of sarcoidosis was confirmed by a biopsy of the scalene nodes. Prednisone $60 \mathrm{mg}$ per day by mouth and $1 \%$ sodium betamethasone eye drops 5 times a day were instituted.

Case 2. A 19-year-old male was diagnosed as having bilateral lymphadenopathy and was referred to our clinic. Vision was 20/20 without correction in both eyes. The applanation pressure was $15 \mathrm{mmHg}$ in the right eye and $14 \mathrm{mmHg}$ in the left. Biomicroscopy showed neither keratic precipitates nor flare in the anterior chamber in both eyes. The iris was normal in appearance in both eyes. Ophthalmoscopy showed nothing abnormal in both eyes. He had complained of no eye symptoms. However, gonioscopy revealed small whitish lesions in the ciliary body band near the iris root in both eyes. Fluorescein gonioangiography (Fig. 2) in the lower nasal quadrant of the right eye revealed shortly after fluorescein injection a 


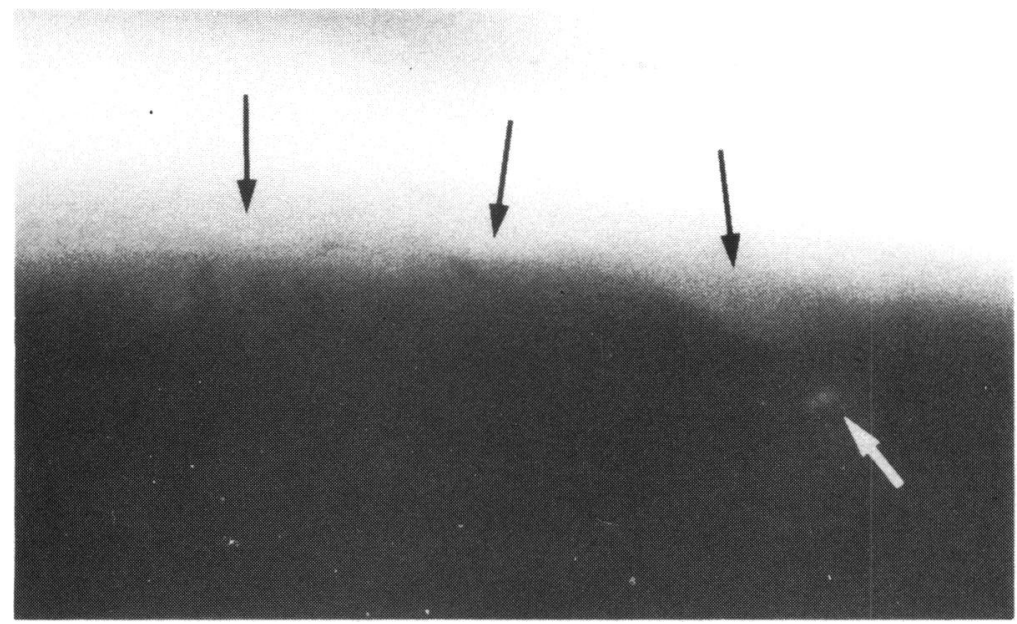

Fig. 1 Fluorescein gonioangiogram in a 21 -vear-old male with ocular sarcoidosis (case 1) showing at 70 seconds a hvperfluorescent dot (white arrow) and relative hyperfluorescence (black arrows) corresponding to the gelatinous nodular lesions in the filtration angle.

hyperfluorescent dot corresponding to the whitish lesion in the ciliary body band. A tuberculin skin test was negative, and the diagnosis of sarcoidosis was confirmed by a biopsy of the conjunctival follicles. $1 \%$ sodium betamethasone instillation 5 times a day was instituted.

Case 3. A 20-year-old male was diagnosed as having bilateral hilar lymphadenopathy and was referred to our clinic. Vision was 20/20 with correction of myopia in both eyes. Biomicroscopy showed neither keratic precipitates nor flare in the anterior chamber in both eyes. The iris was normal in appearance in both eyes. Ophthalmoscopy showed nothing abnormal in both eyes. He had complained of no eye symptoms. However, gonioscopy revealed small whitish lesions in the ciliary body band near the iris root. Fluorescein gonioangiography (Fig. 3) in the lower temporal quadrant of the right eye revealed shortly after fluorescein injection a hyperfluorescent dot corresponding to the whitish lesion in the ciliary body band. A tuberculin skin test was negative, and the diagnosis of sarcoidosis was confirmed by a biopsy of the scalene nodes. $1 \%$ sodium betamethasone instillation 5 times a day was instituted.

Case 4. A 38-year-old male was observed to have enlarged hilar glands on a chest $\mathrm{x}$-ray film and was referred to our clinic. Vision was 20/20 with myopic correction in both eyes. The applanation pressure was $11 \mathrm{mmHg}$ in both eyes. Biomicroscopy showed neither keratic precipitates nor flare in the anterior chamber in both eyes. The iris was normal in appearance in both eyes. He had complained of no eye symptoms. However, gonioscopy revealed several small whitish lesions in the ciliary body band near the iris root. Fluorescein gonioangiography (Fig. 4 ) in the lower nasal quadrant of the right eye

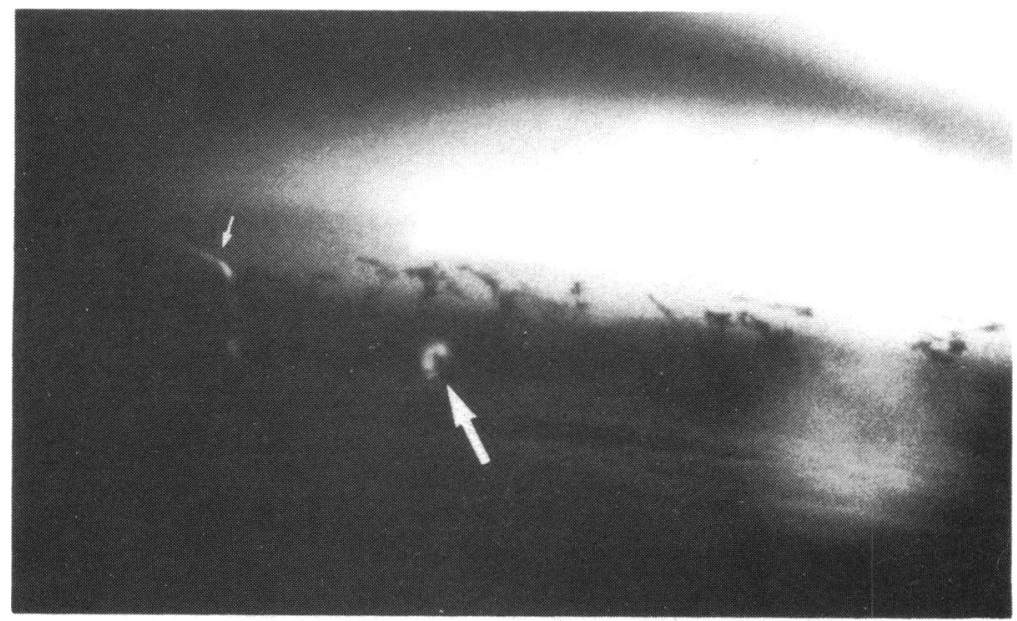

Fig. 2 Fluorescein gonioangiogram in a 19-year-old male with ocular sarcoidosis (case 2) $25 s$ after fluorescein injection showing a hyperfluorescent dot (large white arrow) in the ciliary body band near the iris root. The angle vessel is also fluorescent (small white arrow). 
Fig. 3 Fluorescein

gonioangiogram in a 20-year-old male with ocular sarcoidosis (case 3) $30 \mathrm{~s}$ after fluorescein injection showing a hyperfluorescent dot (white arrow) in the ciliary body band near the iris root.

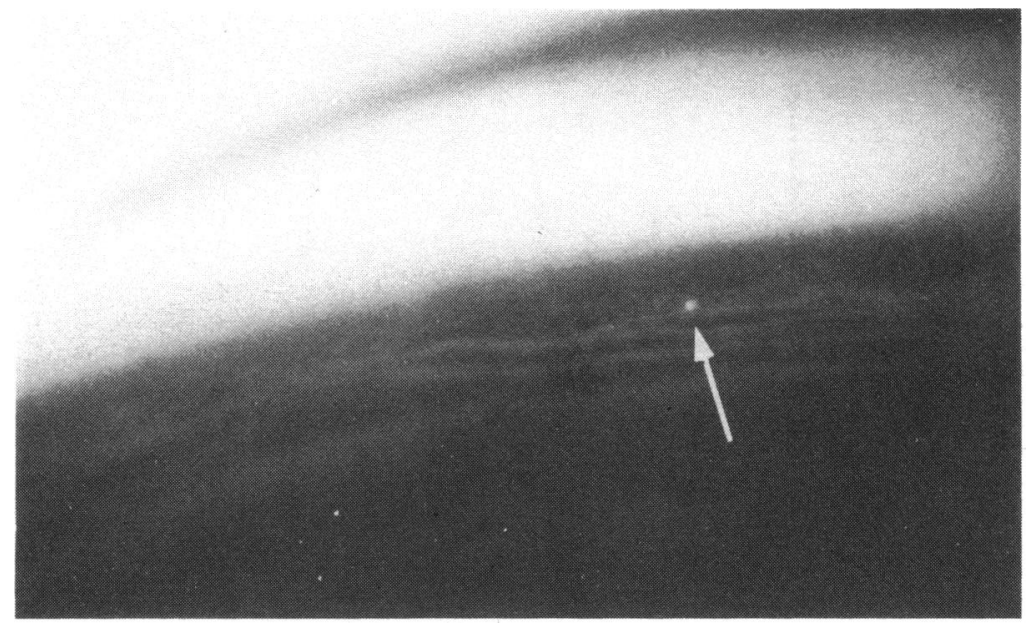

revealed shortly after fluorescein injection hyperfluorescent dots corresponding to the whitish lesions in the ciliary body band. A tuberculin skin test was negative, and the diagnosis of sarcoidosis was confirmed by a biopsy of the scalene nodes. $1 \%$ sodium betamethasone instillation 5 times a day was instituted.

\section{PRESUMPTIVE TUBERCULOUS ANTERIOR UVEITIS} Case 5. A 17-year-old female had complained of blurred vision in both eyes several weeks previously and was seen by an ophthalmologist. She was referred to our clinic with the diagnosis of iritis in both eyes. Vision was 20/20 with myopic correction in both eyes. The applanation pressure was $13 \mathrm{mmHg}$ in the right eye and $16 \mathrm{mmHg}$ in the left. Biomicroscopy revealed not only keratic precipitates but also flare and cells in the anterior chamber in both eyes. The iris was normal in appearance in both eyes. Ophthalmoscopy showed nothing abnormal in both eyes. No abnormal findings were observed on a chest $x$-ray film. A tuberculin skin test was strongly positive. Gonioscopy revealed numerous small whitish lesions in the ciliary body band near the iris root in both eyes. Fluorescein gonioangiography (Fig. 5) in the lower temporal quadrant of the left eye revealed shortly after fluorescein injection numerous hyperfluorescent dots corresponding to the whitish lesions in the ciliary body band. In the late phase of the gonioangiography the ciliary body band was stained. In this case a diagnosis of presumptive tuberculous anterior uveitis was made. Isoniazid $250 \mathrm{mg}$ per day was given by mouth for about 8 weeks, resulting in gradual subsidence of the inflammation.

\section{Discussion}

François ${ }^{3}$ observed both the whitish lesions in the ciliary body band and the gelatinous nodular lesions in the filtration angle in a case of acute iridocyclitis. In his Fig. 1 the whitish lesions in the ciliary body band near the visible edge of the iris are clearly
Fig. 4 Fluorescein gonioangiogram in a 38-year-old male with ocular sarcoidosis (case 4) 20 s after fluorescein injection showing several hyperfluorescent dots (white arrows) in the ciliary body band near the iris root.

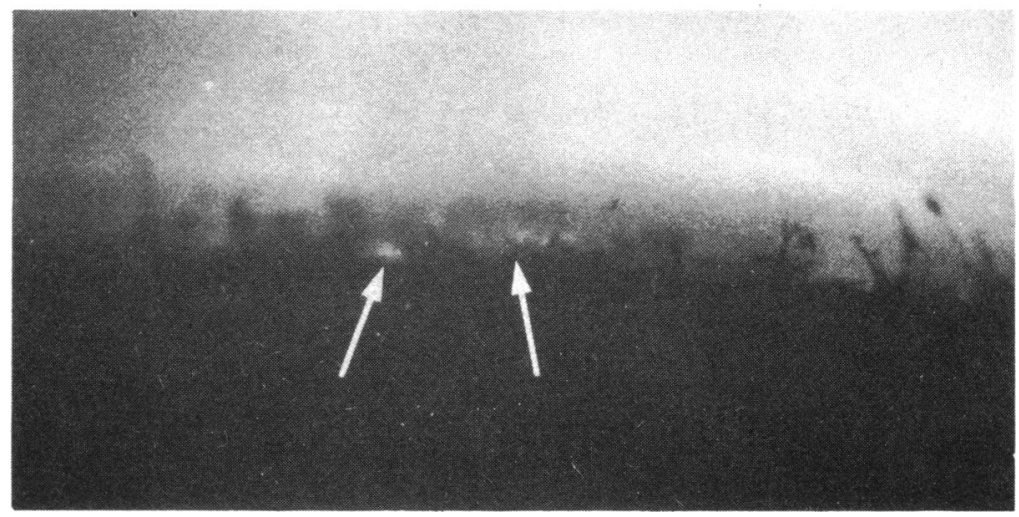




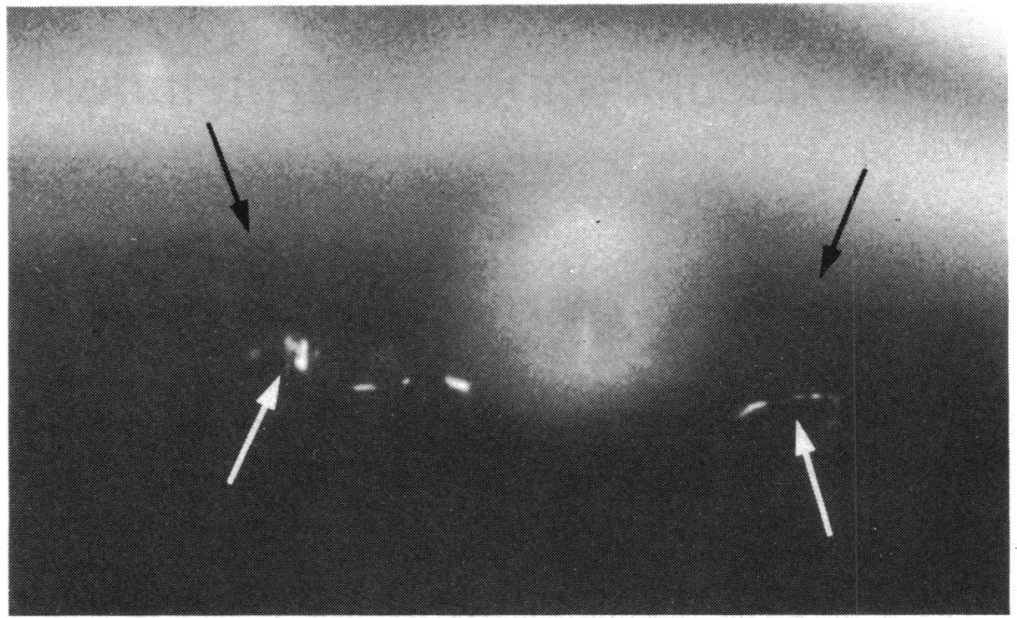

Fig. 5 Fluorescein

gonioangiogram in a 17-year-old female with presumptive tuberculous anterior uveitis, $10 \mathrm{~s}$ after fluorescein injection showing numerous hyperfluorescent dots in the ciliary body band near the iris root. White arrows indicate the iris root and black arrows the scleral spur.

demonstrated. Brockhurst et al. ${ }^{4}$ observed the nodular lesions in the angle in cases of granulomatous uveitis. Because of difficulties in performing tissue biopsies of these lesions in the angle there are few clinicopathological studies of them. Thus the pathogenesis of these lesions in the angle has been a matter of speculation. In the present study by means of fluorescein gonioangiography the whitish lesions in the ciliary body band near the iris root were clearly distinguished from the gelatinous nodular lesions in the filtration angle. The whitish lesions showing hyperfluorescence shortly after fluorescein injection in all 5 cases studied were firmly considered to be granulomas in the ciliary body band. On the other hand the gelatinous nodular lesions in the filtration angle showing relative hyperfluorescence in the late phase of the angiography in case 1 were thought to be not granulomas but exudates. However, further studies by means of gonioscopy, fluorescein gonioangiography, and histological methods should be performed.

In all four cases with ocular sarcoidosis (cases 1-4) studied both the cornea and the aqueous were clear despite apparent changes in the angle. In addition in all 5 cases biomicroscopy revealed neither Koeppe nor Busacca nodules in the iris. In other words, in all cases studied the changes in the ciliary body band preceded that in the iris. As cited by Tronocoso, ${ }^{5}$ Moreu reported in 2 cases of tuberculous uveitis that tuberculomas started in the ciliary body band rather than in the iris. Chandler and Grant $^{6}$ pointed out that in some cases of uveitis there may be no keratic precipitates and no cells in the aqueous but changes in the angle; they have stressed the importance of gonioscopy in such cases. The evidence obtained in the present study strongly supports their suggestion.

\section{References}

1 Eisner G. Biomikroskopische Untersuchungen nach Fluoreszeininjektion. Ophthalmologica 1965; 150: 371-85.

2 Kimura R. Fluorescein goniophotography. Glaucoma 1980; 2: 359-67.

3 François J. Gonioscopic observations during the evolution of an acute iridocyclitis. Am J Ophthalmol 1950; 33: 185-7.

4 Brockhurst RJ, Schepens CL. Okamura ID. Uveitis. I. Gonioscopy. Am J Ophthalmol 1956; 42: 545-54.

5 Troncoso MU. A Treatise on Gonioscopy. Philadelphia: Davis, 1948: 167-8.

6 Chandler PA, Grant WM. Glaucoma. 2nd ed. Philadelphia: Lea and Febiger, 1979: 250. 